\title{
Aproximación a una perspectiva teórica de la Robótica Educativa
}

\author{
Approach to a theoretical perspective of Educational Robotics
}

\author{
Carlos Antonio Vernal Navarrete \\ carlos.vernal.navarrete@gmail.com \\ Código ORCID: 00000-0002-7646-0607 \\ Universidad de Atacama, Copiapó, Chile
}

\author{
Nahur Manuel Meléndez Araya \\ nahur.melendez@uda.cl \\ Código ORCID: 0000-0001-9414-9145 \\ Universidad de Atacama, Copiapó, Chile
}

| Artículo recibido en marzo 2021 | Arbitrado en abril 2021 | Aceptado en abril 2021 | Publicado en mayo 2021

\section{Resumen}

Investigación teórica educativa cuyo objetivo fue comprender los fundamentos teóricos de Robótica Educativa (RE) para aproximar a la comunidad científica docente hacia un planteamiento problemático de estudio con posibilidades de alto nivel, limitaciones para su implementación y discusiones que develan la postura crítica de los investigadores del presente trabajo. En especial desde la visión constructivista. La metodología se orientó a partir del paradigma cualitativo, bajo un enfoque interpretativo de tipo documental. Los resultados abarcan nociones novedosas para repensar la Robótica, las TIC y la enseñanza actual. Se concluyó que la Robótica Educativa representa una opción motivadora que aventaja a los procesos pedagógicos tradicionales centrados en el docente y de impacto para la renovación de discursos tecnológicos.

\footnotetext{
Abstract

Educational theoretical research whose objective was to understand the theoretical foundations of Educational Robotics (ER) to bring the scientific teaching community closer to a problematic study approach with high-level possibilities, limitations for its implementation and discussions that reveal the critical position of the researchers of the present work. Especially from the constructivist perspective. The methodology was oriented from the qualitative paradigm, under a documentary-type interpretive approach. The results include novel notions to rethink Robotics, ICT and current teaching. It was concluded that Educational Robotics represents a motivating option that takes advantage of traditional pedagogical processes focused on the teacher and of impact for the renewal of technological discourses.
}

Palabras clave:

Constructivismo; TIC; Robótica Educativa; fundamentos teóricos; discursos
Keywords:

Constructivism; TIC; Educational Robotics; theoretical fundament; speeches 


\section{INTRODUCCIÓN}

En la actualidad es de suma relevancia las Tecnologías de la Información y Comunicación (TIC) en la educación, siendo de carácter fundamental su utilización en una nueva era basada en la economía del conocimiento (Díaz et al, 2016) (Agencia de calidad, 2018). En este sentido, es pertinente generar espacios de acercamiento a las TIC por su incremento vertiginoso en el hoy. Su sello en diferentes contextos, especialmente el pedagógico, invita a valorar las amplias alternativas de su uso a partir del conocimiento de sus bases que proporcionen un suelo teórico para la investigación educativa en robótica.

En este orden de ideas, se puede inferir que la tecnología ha facilitado el acceso a la información y ha redefinido la forma como las personas nos comunicamos, informamos, aprendemos e incluso pensamos; estos y otros aspectos se han reflejado e integrados en la educación (Brújula, 2018), lo que transforma el aprender en una experiencia conectada. Existen muchos lugares en el mundo para los que el uso de TIC es todavía un lujo, donde habitan personas que nunca han llamado por teléfono (Navarro et al, 2011); éstos son en su mayoría, pertenecientes a zonas rurales de países en desarrollo.

Lo anterior conlleva a generar en campo de las ciencias educativas nuevas interrogantes para estudiar los fundamentos de tecnologías de avanzada. Las tecnologías y su aplicación en la educación se han encontrado hace bastante tiempo, recordando que se ha visto pasar por las aulas, televisores, radios, materiales didácticos, video, etc. Pero en la actualidad nos encontramos con la facilidad de usar diversidad de medios tecnológicos; lo cuales apoyan a capturar la atención de los estudiantes, reducir el tiempo de comprensión, liberar al profesor en tareas repetitivas y sobre todo poner a disposición de quien necesita los contenidos y el conocimiento a unos cuantos clicks de distancia, a través del uso de herramientas sociales (Stirbu, 2015) (Brújula, 2018).

En los últimos años, se ha instalado un creciente interés por incorporar la enseñanza de las Ciencias de la Computación, el Pensamiento Computacional y la Programación en los sistemas educativos (CSTA, 2020) (Rao, 2012). Fundamentado por el aporte de estas temáticas al desarrollo de habilidades de resolución de problemas (Wing, 2010) y de competencias del siglo XXI, tales como: creatividad, pensamiento crítico, colaboración y comunicación (Resnick et al, 2009) (Torrent, 2019); además de ser determinantes para el éxito en una nueva era basada en la economía del conocimiento (Rao, 2012) (Stribu,2015).

Este movimiento a favor de la enseñanza de la computación no es nuevo, ya a finales de los '70 Seymour Papert y el equipo de investigación del MIT con una "tortuga de suelo" programada en Logo, daban impulso a la robótica educativa (RE) (Ratcliffb et al, 2011). Actualmente la robótica forma parte integral de nuestra sociedad expandiéndose a diversas áreas, incluida la educación (Morales, 2017) (García et al, 2012). Sin embargo, aun cuando la $\mathrm{RE}$ promueve interdisciplinariedad, inclusión, interacción, resolución de problemas y aumenta la autoestima de los participantes (Conchinha, 2012) a través de la práctica y experimentación en entornos motivadores (Ribeiro, 2011), son pocas las escuelas que la tienen implementada formalmente en su curriculum, siendo mayoritariamente desarrollada en actividades de carácter extraescolar (Morales, 2017).

Esta investigación tuvo por objetivo comprender los fundamentos teóricos de Robótica Educativa (RE) para aproximar a la comunidad científica docente hacia un planteamiento problemático de estudio con posibilidades de alto nivel, limitaciones para su implementación y discusiones que develan 
la postura crítica de los investigadores del presente trabajo. En especial desde la visión constructivista.

\section{Educación y Constructivismo}

La enseñanza ya no es solo transferir la información del docente a los alumnos, si no crear las oportunidades para construir el conocimiento, bajo esta premisa es que la educación constructivista adopta este cambio conceptual de la educación tradicional.

Para Papert (1982) y Piaget (1980) el mejor aprendizaje no se encontraba en los mejores maestros con las mejores maneras de instruir, si no que estaba en proporcionar al estudiante las mejores oportunidades para construir el conocimiento. Según ellos los estudiantes aprenden mejor cuando se involucran por periodos de tiempo prolongados a construir productos significativos o productos por los cuales tengan un interés mayor. Los proyectos guiados por un maestro, pero abiertos ofrecen generalmente mayores oportunidades para que los estudiantes se involucren, colaboren y contribuyan activamente. Esto permite crear mejores oportunidades para que se instruyan, lo que motivo a Papert y a su equipo a diseñar una variedad de materiales para la construcción de conocimiento para estudiantes, además de ambientes de aprendizaje donde puedan ser utilizados (Harel y Papert, 1991).

Papert (1982) concuerda con Piaget al aseverar que "el constructor necesita materiales para construir" (p.20). Papert afirma que el suministro de la materia prima mediante la cultura puede ser copiosa, escasa o nula para la construcción del conocimiento; sin embargo, en tanto Piaget describe mejor la génesis de la estabilidad mental interna en términos de sucesivas mesetas de equilibrio, Papert expresa que le interesa la dinámica del cambio (Ackermann, 2004).
El conocimiento, para los constructivistas, no es una mera conveniencia que debe transmitirse; entregada en un extremo y codificada, retenida y reaplicada en el otro. Asimismo, el mundo no está solamente sentado allá afuera, esperando ser descubierto, sino que se forma y se reforma progresivamente conforme la gente interactúa con él.

Papert es un fuerte partidario de la idea de que los errores son clave para el aprendizaje, y de que especialmente los(as) niños(as) son expertos a la hora de usar lo poco que saben cómo palanca para crecer (aprendiendo a aprender), Papert ha dedicado buena parte de su vida a la creación de ambientes propiciados por la tecnología, o micro mundos, en los cuales se invita a los aprendices a jugar con ideas que podrían considerarse riesgosas en otro contexto, pero ahora dentro de un espacio seguro.(Ackermann, 2010).

Aplicar esta metodología en las aulas de clases significa un cambio conceptual de la educación tradicional de enseñanza, donde se ven afectados los roles de todos los participantes en el proceso de enseñanza y en el caso del profesor, también significa un cambio en la visión tradicional cediendo el protagonismo al estudiante.

\section{MÉTODO}

La investigación se ubica en el paradigma cualitativo, bajo un enfoque interpretativo de carácter documental, sustentado en la revisión de diversas fuentes bibliográficas vinculadas con el tema de Robótica Educativa. Finaliza con una discusión bibliográfica de aspectos relevantes relacionados con la temática abordada.

Por lo tanto, las exigencias metodológicas de esta investigación establecen una especie de vínculo implícito con el problema de investigación, porque en la medida que se lleve a cabo el examen profundo de los ejes interpretativo, argumentativo $y$ escritural 
(propios de una investigación teórica), se comprenderán conceptos, teorías y visiones críticas que complejizarán el tejido de relaciones entre todos los elementos que constituyen el objeto de estudio, que serán el aporte de una perspectiva teórica sobre la robótica educativa.

Ahora bien, ¿cuáles serían esos elementos teóricos y categorías que conformarán ese tejido particular del objeto teórico a construir por el investigador?

Los planteamientos de la tecnología de la información (TIC's) enunciados en el panorama teórico educativo conformarán el tejido central de una producción de conocimientos que develará una perspectiva que involucra, relaciona a éstos con la formación.

\section{RESULTADOS}

A partir del análisis de los documentos recopilados se obtuvieron un conjunto de visiones producto del proceso hermenéutico de la investigación, los cuales condujeron a las nociones del rol del docente; TIC's; Educación robótica y Actividades propias de la RE, las cuales no escapan a un paradigma educativo constructivista definido por el docente y el estudiante.

\section{Rol del Docente}

Autores como Barrera en (Barrera, 2014) y Contreras en (Aliaga et al, 2018) recalcan la importancia del rol que juega el docente constructivista, en un principio dicho rol es de mediador, pero a medida que transcurre el proceso el rol del docente se transforma en el de facilitador. La enseñanza centrada en el docente se sustituye por la asistencia al estudiante en sus descubrimientos a través de construcciones que le permitan comprender y entender problemas de manera práctica. En ese sentido, el desarrollo de competencias básicas investigativas conlleva la explicación del mundo físico por medio del diseño y construcción de prototipos tecnológicos para resolver problemas de su entorno. El estudiante explora utilizando métodos científicos a situaciones, describe, explica los factores de la realidad en base a postulados científicos. Asimismo, al encontrarse con situaciones problemáticas de la realidad, ensaya el diseño y producción de modelos que a la postre constituirán prototipos tecnológicos para construir alternativas tecnológicas en aras de mejora de la calidad de vida.

En cuanto a la formación de los docentes de escuelas multigrado según Ames (2004) las estrategias de capacitación han seguido diversos modelos (en cascada, con equipos multiplicadores, con microcentros o centros demostrativos) siendo algunas de esas experiencias más exitosas que otras. En todos los casos, se encuentra un énfasis en partir del aula y volver a ella, de manera que esto permita a los docentes modificar efectivamente sus prácticas pedagógicas. Al enfocarse en el aprendizaje por descubrimiento se busca que el estudiante logre conocer a través de un razonamiento inductivo. Es decir, pasar de lo particular (detalles y ejemplos) a lo general (principios o conceptos). Así como también, requiere del involucramiento del estudiante en su propio proceso de aprendizaje. Es necesaria la participación activa de los estudiantes en el proceso de aprendizaje, donde estudiante y docente cooperan en la resolución del problema (Willging et al, 2017).

Piaget aplico su modelo (constructivista) a problemas de acción y observo como los niños resuelven problemas explorando el mundo mediante acciones concretas. El aprendizaje constructivista está basado en el equilibrio/ asimilación por parte de los estudiantes. Algo que noto fue que los estudiantes al enfrentarse a un nuevo problema, similar a otros que saben resolver, pero con cambios sutiles para diferenciarlos, estos se desequilibran, es ahí donde el rol del docente debe ser resolver el desequilibrio y que resulte en un aprendizaje significativo para ellos (Pina, 2017). 
Se espera de un estudiante que sea capaz de aprovechar responsablemente el uso de las TIC's para interactuar con la información, gestionar su comunicación y aprendizaje. En el MINEDU (MINEDU, 2017) definen esta competencia en cuatro capacidades que los estudiantes deben tener en su curriculum:

- Personaliza entornos virtuales.

- Gestiona información del entorno virtual.

- Interactúa en entornos virtuales.

- Crea objetos virtuales en diversos formatos.

Para facilitar que sus estudiantes logren el perfil de egreso, el docente debe desarrollar progresivamente competencias que no necesariamente recibió en su formación inicial.

\section{Robótica Educativa}

La Robótica Educativa (RE) no es simplemente montar robots, sino que los estudiantes tienen que seguir indicaciones para poder juntos, decidir cuál es la mejor manera para construir ese robot y programarlo con el objetivo de resolver el problema que se les ha presentado (Morales, p, 2017). Sin embargo, tal como se plantea en (Suárez et al, 2018), no se puede suponer que el uso de la tecnología por sí sola mejorará de forma automática los aprendizajes. En este aspecto, aun cuando introducir e implementar la robótica en las escuelas proporciona mejoras en la creatividad e imaginación de los niños, aprenden a dar soluciones innovadoras y les permite trabajar en equipo, son pocas las escuelas que la tienen implementada formalmente en su curriculum, siendo mayoritariamente desarrollada en actividades de carácter extraescolar (Morales, 2017).

La educación es el recurso clave en un mundo en el que la fuente de poder y riqueza es la capacidad de procesar información para transformarla en conocimiento aplicado
(Cuevas et al, 2009) (Meléndez et al, 2017). Se busca una formación de ciudadanía digital a través de una comprensión crítica del mundo actual (Borchardt et al, 2017), fomentando una participación activa, no sólo como simples "consumidores/usuarios de tecnologías", sino como entes "creadores con/de tecnologías" (Kafai, 2016) (Hitschfeld et al, 2015). Es así que las TIC se han convertido en una herramienta fundamental en este nuevo proceso (Meléndez et al, 2017), pero no se puede suponer que el uso de la tecnología por sí sola mejorará de forma automática el aprendizaje (Orozco, 2016). Es así que las nuevas generaciones están siendo partícipes del surgimiento de una cultura DIY/ HUM (Do It Yourself - Hágalo Usted Mismo), que arraiga un compartimiento particular relacionado con la creación con/de tecnología, donde más allá de saber programar, el crear y compartir implica indudablemente el conectarse y colaborar con otros (Torrent, 2019) (Kafai, 2016).

El origen de la Robótica Educativa no es del todo claro, algunos autores (Castro et al,2017) le atribuyen su origen en la teoría constructivista de Piaget, en cambio (Morales et al, 2017) arroga su origen en el construccionismo de Papert, quien aplico el constructivismo de Piaget a las TIC, Aun así consideran a la Robótica Educativa como una herramienta de aprendizaje muy versátil y polivalente que permite trabajar en diferentes áreas del conocimiento propiciando la adquisición de diversas habilidades. También están de acuerdo en el potencial que tiene, ya que permite que los alumnos mantengan la atención y percepción al poder integrar lo teórico y la realidad por medio de la actividad, el niño avanza en su didáctica como si de un juego se tratase.

Ruiz Velasco en (Velasco, 2007), la define como "una disciplina que permite concebir, diseñar y desarrollar robots educativos para que los estudiantes se inicien desde muy jóvenes en 
el estudio de las Ciencias y la Tecnología” (s.n.). Según Willging y colegas (Willging et al, 2017), "La robótica se refiere al diseño y aplicación de robots, que son máquinas que realizan una serie de tareas automatizadas" (s.n.) "La robótica forma parte de un enfoque pedagógico centrado en el alumno, que le permite construir objetos tangibles de su propio diseño y con sentido para él." (s.n.), es así una disciplina que genera entornos de aprendizaje heurístico poniendo el foco en la participación activa de los estudiantes, quienes construyen sus aprendizajes a partir de su experiencia durante el proceso de construcción y programación de los robots. La robótica educativa entonces, convierte a la robótica en un medio para alcanzar ciertos aprendizajes.

En este contexto, lo importante no es conseguir expertos en robótica, ni se busca desarrollar en los estudiantes, competencias en automatización industrial y control automático de procesos (Morales, 2017) (Barrera, 2015). Sino que se busca hacer de la robótica una excusa para comprender, hacer y aprehender la realidad (Barrera, 2015). Es así que la construcción misma de un Robot educativo, es un excelente pretexto para lograr esta integración desde el punto de vista cognitivo y tecnológico (García et al, 2017).

Barrera en (Barrera, 2014), plantea que la robótica educativa (RE) tiene por objeto poner en juego toda la capacidad de exploración y de manipulación del sujeto cognoscente al servicio de la construcción de significados a partir de su propia experiencia educativa. También sugiere el uso de la robótica educativa como andamiaje instrumental, haciendo posible integrar $y$ materializar algunos de los saberes abordados en otras áreas de formación de la educación básica y/o media, por ejemplo, la construcción misma de un robot educativo requiere conocimiento de diversas áreas y es un excelente pretexto para lograr la integración desde el punto de vista cognitivo y tecnológico (García et al, 2017).
La robótica educativa representa una opción motivadora que aventaja a los procesos pedagógicos tradicionales centrados en el docente; hace que las asignaturas sean más atractivas y fáciles de trabajar cambiando el enfoque hacia los estudiantes. Por lo que constituye una nueva alternativa para la labor docente, impulsando las disciplinas en las distintas áreas (Ovalles et al, 2018). Este enfoque centrado el en estudiante permite la construcción de objetos tangibles de su propio diseño y con un sentido para él, generando entornos de aprendizajes heurísticos, poniendo el foco en la participación activa de los estudiantes, donde los aprendizajes se construyen a partir de las experiencias del proceso de diseño, construcción y programación de los robots. La RE entonces, convierte a la robótica en un medio para alcanzar ciertos aprendizajes (Willging et al, 2017).

Cabe mencionar el reto, tal como se ha mencionado, consiste en utilizar la robótica como herramienta para aprender de otras áreas y como el fin, tan solo como un medio (Pina, 2017). Para ello debemos adaptar las actividades y competencias claves que componen el currículo de los estudiantes. Morales en (Morales et al, 2017) se habla de la importancia de no formar expertos en robótica, si no en generar ambientes de aprendizajes en los que los estudiantes puedan poner en marcha sus ideas utilizando su imaginación y creatividad, que aprendan a solucionar problemas y trabajen de manera colaborativa. Así aumentar su motivación para que consigan un aprendizaje mayor tanto en cantidad como calidad.

\section{Actividades de Robótica Educativa}

Autores como Pina en (Pina, 2017) y Castro en (Castro et al, 2017) describen la RE como un medio y no como un fin, y remarcan su importancia en la educación de los nativos digitales. Las actividades de RE comogeneradores de entornos de aprendizajes permiten involucrar 
a los estudiantes en actividades lúdicas con robots educativos, estas actividades tienen como objetivo educar y contribuir al desarrollo del alumno, estimular y potenciar sus aptitudes a través de la creatividad, la autonomía personal, las relaciones entre los alumnos, el trabajo en equipo, la cooperación, etc (Castro et al, 2017).

Es fundamental recalcar que el carácter lúdico de la actividad, en un ambiente desinhibido, cordial, amigable y ocioso favorece el aprendizaje. En este sentido, el juego funciona como una estrategia didáctica (Pizarro, 2007) de forma que los alumnos olvidan que están siendo observados y abandonan la timidez y las dificultades, haciendo que aprendan el contenido propuesto más fácilmente, buscando que desarrollen conceptualizaciones que les permitan abordar problemas cotidianos relacionados con el adecuado uso de la tecnología, ya que estos saberes se hacen indispensables en la interacción sociocultural y en la interacción con el entorno natural de los ciudadanos del siglo XXI (Barrera,2014).

A través de las actividades de RE se busca también motivar mediante el estímulo de la curiosidad científica de los sujetos, la indagación, experimentación y construcción de saberes que disminuyan la distancia existente entre el conocimiento científico y los saberes usados por las personas en la cotidianidad (Barrera,2014). De esta forma no solo trata de despertar vocaciones científico-técnicas, si no, al establecerse como medio de acción disponible del proceso educativo, por el carácter activo, participativo y cooperativo de los estudiantes busca desarrollar habilidades y competencias en los alumnos a través del juego. (Castro et al, 2017).

Con los robots, podemos extender la aplicación del modelo constructivista a problemas de formulación donde los estudiantes pueden resolver problemas mediante la exploración de formulaciones hipotéticas del mundo. Se desarrolla, así, el pensamiento "hipotético-deductivo" (Pina, 2017).

\section{DISCUSIÓN}

Durante la investigación se realizaron consultas bibliográficas en bases de datos científicas entre los cuales se destacan los siguientes fragmentos:

Con respecto a la Robótica Educativa (RE) el autor plantea en (Barrera, 2014) que la investigación de RE nace de una necesidad emergente en la base de la comunidad académica, como resultado de la permanente tensión dialéctica que sostienen la teoría y la práctica educativa, como lo señala Moreno (Moreno, 2005). Es así como surgen ideas que, aunque están lejos de eliminar las dificultades al interior de la escuela, representan un pequeño y moderado paso en la búsqueda de tal fin. Entonces nace como propuesta la creación de ambientes para la enseñanza, de carácter lúdico, empleando «robots educativos» como instrumentos didácticos, creando rutas que permitan abordar el conocimiento como un todo, carente de fragmentos, utilizando como derrotero las teorías del aprendizaje y el construccionismo de Papert y, así, crear nuevas estrategias que den como resultado la mejora de los procesos de enseñanza/aprendizaje.

No obstante, se debe resaltar que la Robótica Educativa, como herramienta que apoya los procesos de enseñanza-aprendizaje desde la perspectiva educativa, toma la dimensión de medio y no de fin. No se busca que los estudiantes adquieran competencias en automatización industrial y control automático de procesos, solo se busca hacer de la robótica una excusa para comprender, hacer y aprehender la realidad (Barrera, 2014).

Al mismo tiempo Pina (2017) menciona que las necesidades actuales (y futuras) de nuestro mercado laboral en el sector de la $\mathrm{I}+\mathrm{D}+\mathrm{i}$ hacen que la Educación en Ciencia y Tecnología 
sea desde hace unos cuantos años un objetivo prioritario en las administraciones y Sociedad Europeas. Esta necesidad se ha ido reflejando en Universidades y Educación Secundaria y debe estar también presente en Educación Primaria, y de hecho empieza a estarlo. En cuanto a su aplicación en al aula Pina hace mención a su aplicación en el aula y como surgen una serie de problemas como son la necesidad de compra de material robótico, la necesidad de formación y apoyo del profesorado y la organización en el aula. Son aspectos que se van resolviendo de diferentes maneras en cada centro (organizaciones horarias diferentes, con equipos de profesores, con servicios de préstamos de equipos o con cursos de formación del profesorado organizados por las administraciones educativas).

Así el autor antes mencionado también acentúa la importancia de involucrar a la sociedad en general en la educación, y en particular en la educación de Ciencia y tecnología. Para ello es importante la implicación de las familias y de la propia sociedad. Es necesario crear y apoyar diferentes actividades de formación en Ciencia y tecnología, fuera de la escuela, como pueden ser torneos y competiciones robóticas, o campamentos y clubs tecnológicos. En este sentido es de destacar la implicación que están teniendo en estas actividades instituciones como Museos o Planetarios o entidades que promueven la innovación, creatividad y el emprendimiento.

Debemos recordar que el siglo XXI es el propio de la información y la comunicación y a los nuevos trabajadores que traerá este siglo se les va a exigir que sepan plantear soluciones innovadoras a problemas existentes (Morales et al, 2017).

Cada fundamento teórica abre perspectivas para repensar la aplicación de la Robótica Educativa en la actualidad, pues es un área del saber que tiene por finalidad configurar nuevos espacios de aprendizaje sustentados en generar otras didácticas donde el protagonista es el estudiante y los problemas a solucionar.

\section{CONCLUSIONES}

En este estudio se logró concluir que la robótica educativa representa una opción motivadora que aventaja a los procesos pedagógicos tradicionales centrados en el docente; hace que las asignaturas sean más atractivas y fáciles de trabajar cambiando el enfoque hacia los estudiantes generando ambientes de aprendizaje en los que los estudiantes puedan poner en marcha sus ideas utilizando su imaginación y creatividad, que aprendan a solucionar problemas y trabajen de manera colaborativa. Por lo que constituye una nueva alternativa para la labor docente, impulsando las disciplinas en las distintas áreas $y$ otros discursos educativos que promuevan transformación críticas.

Interpretar las posturas críticas teóricas para redactar una aproximación esta íntimamente vinculado con la noción esencial de la robótica de avance y desarrollo. Progreso que impulsa a la Educación a transformar discursos para cosechar motivaciones en las comunidades científicas de alto impacto tecnológico y social.

\section{REFERENCIAS}

Ames, P. (2004). "Las escuelas multigrado en el contexto educativo actual: desafíos y posibilidades". Ministerio de Educación del Perú. Recuperado el 8 de agosto de 2020 de: https://www.academia.edu/28137157/ Las_escuelas_multigrado_en_el_contexto_ educativo_actual_desaf\%C3\%ADos_y_ posibilidades

Ackermann, E. (2001). Piaget's Constructivism, Papert's Constructionism: What's the difference? En Future of Learning Group Publication, 5 (3). Recuperado de: http://learning.media.mit.edu/content/ publications/ EA.Piaget\%20_\%20Papert.pdf Agencia de Calidad de la Educación, "Los Desafíos para la Nueva Educación Rural", Chile, 2018, recuperado el 8 de agosto de 2020 de: https://url2.cl/AhCbS 
Aliaga Contreras, I., Carhuaricra Cusipuma, J., Asencios Trujillo, L., \& Piñas Rivera, L. (2018). "Programa de robótica educativa para mejorar el aprendizaje significativo en estudiantes del cuarto grado del área de Ciencia y Ambiente de la institución educativa San Roque - Castrovirreyna", 2015. Revista EDUCA UMCH, 11(11), pág. 147-161

Barrera-Lombana. N. (2014). "Uso de la robótica educativa como estrategia didáctica en el aula” Praxis \& Saber, Vol. 6. Núm. 11, pp. 215-234

Borchardt, M. Roggi, I. "Ciencias de la Computación en los sistemas educativos de América Latina”. Cuaderno SITEAL/ TIC Sistema de Información de Sistemas Educativos de Latinoamérica. UNESCOOEI. Enero 2017. Buenos Aires, Argentina. Recuperado el 8 de agosto de 2020 de: https:// url2.cl/RfwLk

Brújula Investigación y estrategia informe final, Chile (2018). "IX Encuesta de Acceso y Usos de Internet". Recuperado de https://www. subtel.gob.cl/estudios/internet-y-sociedadde-la-informacion/

Castro, F., Briegas, J. J., González, S., \& González, D. (2017). "Actividad extraescolar para aprender a aprender: la robótica como herramienta educativa”. Revista De Estudios E Investigación En Psicología Y Educación, (13), 124-128. https://doi.org/10.17979/ reipe.2017.0.13.2542

Conchinha, C., "Lego Mindstorms: Um estudo com utentes com paralisia cerebral". Atas do II Congresso Internacional TIC e Educação (2012), pp. 1581-1593

Cuevas, P., Pacheco E, P., Rodríguez G., Santos A, A., "Tecnologías de Información y comunicación (TICs) en la Educación Superior". Universidad Técnica Particular de Loja, 2009

CSTA, "K-12 Computer Science Standards, Revised 2017". Association for Computing Machinery, Code.org, Computer Science Teachers Association, Cyber Innovation Center, and National Math and Science. New York, 2017. Recuperado el 8 de agosto de 2020 de: https://url2.cl/d1URg
Díaz, R., Osses, S., Muñoz, S., "Factores e interacciones del proceso de enseñanzaaprendizaje en contextos rurales de la Araucanía, Chile", revista Estudios pedagógicos vol.42 no.3, Valdivia, Chile, 2016

Fernández Morales.C., Iriarte Gómez. F., Mejía Solano.C., Revuelta Domínguez.F., (2018) "contextualización de la formación virtual en robótica educativa de los docentes rurales del Perú" Revista de Estudios y Experiencias en Educación Número Especial No 3, junio 2018 pp. $71-82$

García, M., Castillo, L. y Escobar A., "Plataforma robótica educativa ROBI", Revista Colombiana de Tecnologías de Avanzada, 2012, 19(1)

Harel, I. and Papert, S. (Eds) (1991). Constructionism. Norwood, NJ. Ablex Publishing Co

Ince, N. B., \& Sahin, A. E. (2016). "A Comparison of Combination Classroom Teachers' and Single-Grade Teachers' Job Satisfaction and Burnout Levels". Hacettepe Universitesi Egitim Fakultesi Dergisi-Hacettepe University Journal of Education, 31(2), 391409

Kafai, Y. "From Computational Thinking to Computational Participation in K-12 Education". Communications of the ACM, August 2016, pp. 26-27. Recuperado el 8 de agosto 2020 de: https://doi. org/10.1145/2955114

Meléndez Araya, N.M., "Incorporation of initial research skills in undergraduate engineering", 36th International Conference of the Chilean Computer Science Society (SCCC), Arica-Chile (2017)

Meléndez Araya, N.M., Cortes Cabrera, M.A., "Application to use of learning objects in higher education", 36th International Conference of the Chilean Computer Science Society (SCCC), Arica-Chile (2017)

MINEDU. (2017). "Currículo Nacional de la Educación Básica (Aprobado mediante Resolución Ministerial Nro. 281-2016-ED Modificado mediante RM Nro. 159-2017ED)”. Recuperado el 8 de agosto de 2020 de: 
http://www.minedu.gob.pe/curriculo/pdf/ curriculo-nacional-2017.pdf

Morales, P., "La robótica educativa: una oportunidad para la cooperación en las aulas", Innovación docente y uso de las TIC en educación, 2017, pp. 124-131

Navarro, I., Pertegal, M.L., Gil, D., González, C., Jimeno, A., "El aprendizaje basado en proyectos como estrategia didáctica $\mathrm{y}$ pedagógica para estimular el desarrollo de competencias profesionales". IX Jornades de Xarxes d'Investigació en Docència Universitària. Universitat d'Alicante, 2011

Ovalles Germosén. A., Luna Tejada. R.E., Pérez Teruel. K. (2018) "Modelo pedagógico con la robótica educativa como apoyo didáctico en la enseñanza de matemática de primaria", Educación Superior Año XVII No. 25. Enero-Junio, 2018 pp. 011 a 029

Papert, S. (1982). Desafío a la mente: Computadoras y educación. Buenos Aires: Galápago

Piaget, J. (1980). Psicología y pedagogía. Barcelona: Ariel

Pina-Calafi, A. (2017). "Robótica Educativa en Educación Primaria: ¿por qué y cómo?” En S. PérezAldeguer, G. Castellano-Pérez, y A. Pina-Calafi (Coords.), Propuestas de Innovación Educativa en la Sociedad de la Información (pp. 15-27). Eindhoven, NL: Adaya Press

Rao, M. (Ed.). "Knowledge management tools and techniques". Practitioners and Experts Evaluate KM Solutions. London: Routledge, 2012. (J2 y J5)

Ratcliffb, C. C. \& Andersona, S. E., "Reviving the turtle: Exploring the use of logo with students with mild disabilities". Computers in the Schools, Septiembre 2011, pp. 241-255 Ribeiro, C, Coutinho, C., \& Costa, M., "Robowiki:
Um recurso para a robótica educativa em língua portuguesa”. VII Conferência Internacional de TIC na Educação, 2011, pp. 1499-1514

Stirbu, O. A., "Theoretical approach regarding the competitiveness of SMEs". International Journal of Advanced Research, 2015, 3 (6): pp.1057-1063

Suárez Zapata. A., García Costa. D., Martínez Delgado. P., Martos Torres. J., "Contribución de la robótica educativa en la adquisición de conocimientos de matemáticas en la Educación Primaria" Revista de Formación del Profesorado e Investigación Educativa, Universidad de Oviedo, pp. 43-54, Enero Diciembre, 2018

Torrent, C., (2019). "Programación computacional en escuelas: una mirada de inclusión y género a una iniciativa extracurricular del Ministerio de Educación de Chile", Universidad de Chile, SantiagoChile

Velasco, E.R.,(2007).“Educatrónica: Innovación en el aprendizaje de las ciencias y la tecnología", Madrid: Díaz de Santos

Willging P.A., Astudillo G.J., Castro L., Bast S., Occelli M., Distel J.M., "Educación con tecnologías: la robótica educativa aplicada para el aprendizaje de la programación", 2017, pp. 1174-1178, recuperado el 8 de agosto de 2020 de: http://sedici.unlp.edu.ar/ handle/10915/62859 